\title{
Compressive Sensing on Diverse STEM Scans: Real-time Feedback, Low-dose and Dynamic Range
}

\author{
Xin Li, ${ }^{1,2}$ Ondrej Dyck, ${ }^{1,2}$ Sergei V. Kalinin, ${ }^{1,2}$ and Stephen Jesse, ${ }^{1,2}$ \\ ${ }^{1 .}$ Center for Nanophase Materials Science, Oak Ridge National Laboratory, Oak Ridge, TN, USA. \\ 2. Institute for Functional Imaging of Materials, Oak Ridge National Laboratory, Oak Ridge, TN, USA.
}

Scanning transmission electron microscopy has become the main platform to analyze materials at atomic resolution. Recently, the STEM community is rapidly trending towards exploring beam induced chemical processes and especially manipulating atomic motion to enable atom-by-atom fabrication $[1,2,3]$. These newly emerged beam fabrication applications, together with traditional imaging of beam sensitive materials, bring forward challenges of reducing electron dose and increasing the absolute scanning speed, which will act to decrease the likelihood of unintentional beam-induced modification of the structure being fabricated and enable real-time feedback.

A promising route for low-dose imaging is the compressed sensing method that was broadly introduced to STEM community by Stevens and Browning $[4,5]$ that mainly focus on the random sub-sampling. However, random sub-sampling approach may have limited effects on reducing the absolute scanning duration, since it takes time for the beam to move between random positions. Finding the shortest path connecting randomly located scanning sites is NP-hard, known as the travelling sales man problem. A recent study [6] investigated several hardware-friendly scan pathways with smooth, continuous, and ideally single frequency input voltage waveform such as spiral scans, trying to cover the scanning area with minimum beam movement distance and time, yielding sparsely sampled images at video rate. Scans with continuous driven voltages give users direct control on the distribution of electron dose. For example, the amplitude of driven voltage determines the physical scanning area, increasing frequency of driven voltage will increase the spatial denseness of electron distribution meanwhile increasing scanning duration will improve average electron density and thus the signal to noise ratio.

Real-time (milliseconds) image reconstruction from fast and sparse scans can be achieved by the GPU implementation (opensource code available online [6,7]) of the iterative soft-thresholding algorithm on the wavelet transform basis [8,9]. Figure 1 illustrates some examples of sparse and diverse scanning pathways with reconstruction results.

To probe limits of the inpainting algorithm, various scanning configurations was conducted in [6]. It turns out that sparseness and unevenness in sampling density have synergistical effects on the success of reconstruction: neither the scanning frequency nor the scanning duration can be too low. It is important to note that increasing frequency (reducing the sparseness) does not necessarily compensate the negative impacts introduced by decreasing scanning duration, and vice versa. Future efforts can be made on establishing a systematical optimization model to derive "optimal" scanning pathways where successful reconstruction can be made, given hardware and tasks-customized constraints (such as voltage, scanning area, scanning duration) [10].

References:

[1] O Dyck et al., Nano Research 11 (12) (2018), p. 6217.

[2] O Dyck et al., Small 14 (38) (2018), p. 1801771. 
[3] T Susi et al., 2D Materials 4 (4) (2017), p. 042004.

[4] A Stevens et al., Microscopy 63 (1) (2013), p. 41.

[5] A Stevens et al., Advanced Structural and Chemical Imaging 1 (1) (2015), p. 10.

[6] X Li et al., Microscopy and Microanalysis 24 (6) (2018), p. 623.

[7] X Li, RTSSTEM package, https://github.com/nonmin/RTSSTEM

[8] I Daubechies et al., Communications on Pure and Applied Mathematics 57 (11) (2004).

[9] G Peyré, Computing in Science \& Engineering 13 (4) (2011), p. 94.

[10] Research was supported by the US Department of Energy (DOE), Office of Science, Basic Energy Sciences (BES), Materials Sciences and Engineering Division and Office of Science User Facility and was performed at the Center for Nanophase Materials Sciences at Oak Ridge

National Laboratory. We gratefully acknowledge the support of NVIDIA Corporation with the donation of the Titan X Pascal GPU used for this research.
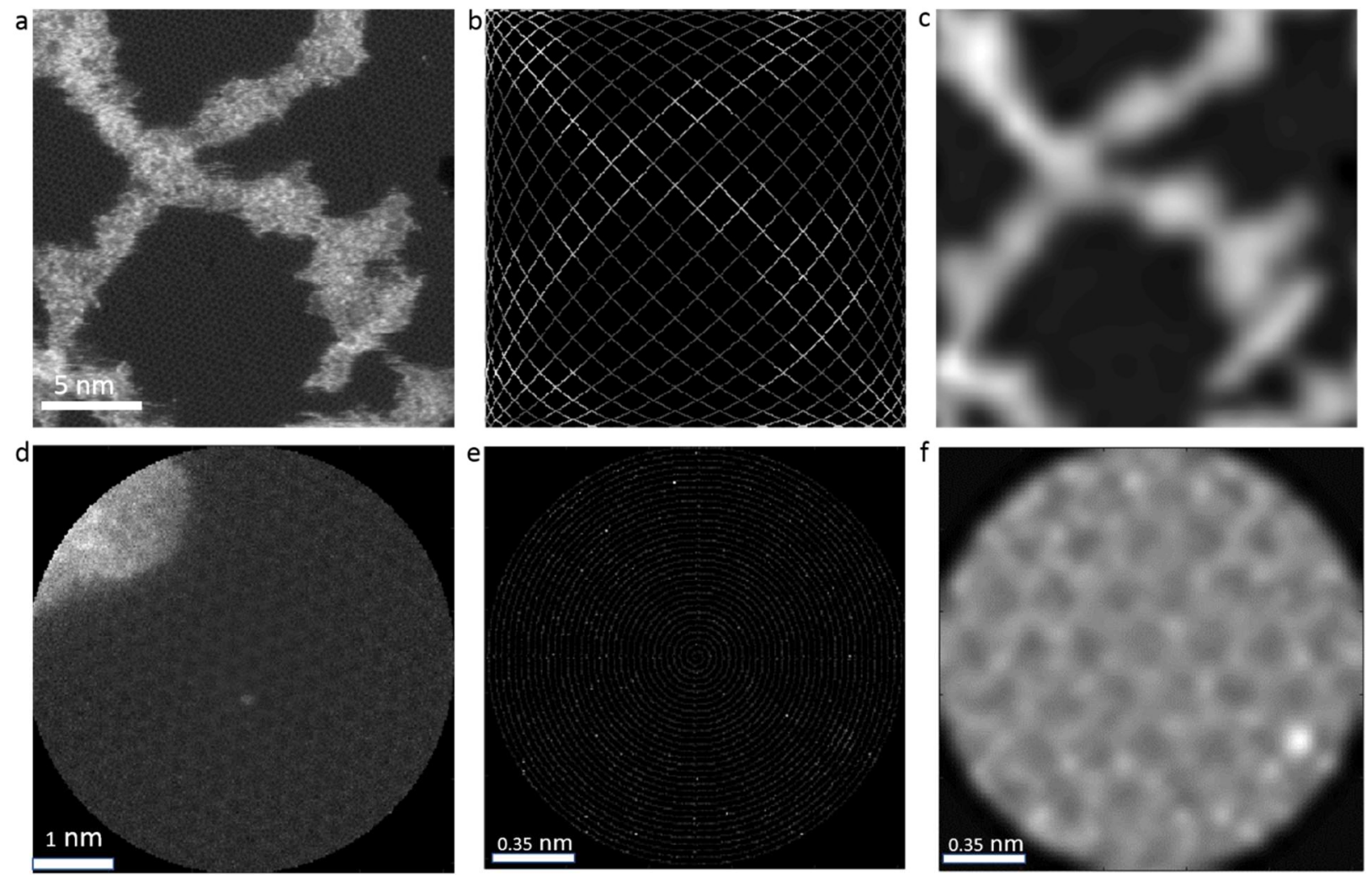

Figure 1. a) Regularly scanned image over the graphene with large dwell time at a low magnification level, as the reference. b) A synthetic sparse Lissajous scan over the reference image. c) Reconstructed image in real-time from the sparse Lissajous scan. d) A dense spiral scan over the graphene at a low magnification level, with a large frequency of $256 \mathrm{~Hz}$ and total scanning duration of 6.4 seconds. e) A sparse spiral scan over the graphene at a high magnification level, with frequency of $32 \mathrm{~Hz}$ and total duration of 0.8 seconds. f) Reconstructed image in real-time (millisecond) from the b) sparse spiral scan. 